\title{
THE BOUNDARY OF THE NUMERICAL RANGE
}

\author{
JOHN S.' LANCASTER 1
}

ABSTRACT. This work includes two result's which indicate a set theoretic relationship between the boundary of the numerical range and the essential numerical range. Several applications are derived.

Let $\mathcal{H}$ be a separable, infinite dimensional complex Hilbert space with inner product (,), and let $\mathscr{B}(\mathcal{H})$ denote the algebra of bounded linear operators on $\mathcal{H}$. The numerical range of $T \in \mathcal{B}(\mathcal{H})$ is by definition the set

$$
W(T)=\{(T x, x): x \in \mathcal{H},\|x\|=1\} .
$$

It is well known that $W(T)$ is a bounded (not necessarily closed) convex subset of the plane. Furthermore, the closure of $W(T)$, denoted $W(T)^{-}$, contains the spectrum $\sigma(T)$ of $T$ (see [4]).

The essential numerical range of $T$ can be defined as the set

$$
W_{e}(T)=\bigcap\left\{W(T+K)^{-}: K \in \mathcal{K}\right\},
$$

where $\mathcal{K}$ denotes the ideal of compact operators on $\mathcal{H}$. Clearly $W_{\boldsymbol{e}}(T)$ is a compact convex subset of the plane and $W_{e}(T) \subset W(T)^{-}$. It is known that $W_{e}(T)$ contains the essential spectrum $\sigma_{e}(T)$, which can be viewed as the spectrum of $T$ represented canonically in the quotient $\mathcal{B}(\mathcal{H}) / \mathcal{K}$ (see [9]).

This paper is concerned with relationships among the sets $W(T)$, $W(T)^{-}$, and $W_{e}(T)$. The following characterization, due to P. Fillmore, J. G. Stampfli, and J. P. Williams [2], is of fundamental importance: $\lambda \in W_{e}(T)$ if and only if there exists a sequence $\left\{x_{n}\right\}_{1}^{\infty}$ of unit vectors in $\mathcal{H}$ such that $x_{n} \rightarrow 0$ weakly and $\left(T x_{n}, x_{n}\right) \rightarrow \lambda$.

In what follows, Ext $(C)$ denotes the set of extreme points of a convex

Presented to the Society, January 17, 1972; received by the editors June 25, 1973. 47A55.

AMS (MOS) subject classifications (1970). Primary 47A10, 47B10; Secondary

Key words and phrases. Operator on Hilbert space, numerical range, essential numerical range, extreme point of a convex set, compact operator.

1 Part of this work appears in the author's doctoral dissertation (Indiana University, May, 1972), which was directed by J. P. Williams and supported by an NSF Traineeship. The remainder was done with support of the Marshall University Research Fund. 
set $C$, convh $(X)$ denotes the convex hull of the set $X$, and $\partial X$ denotes the topological boundary of. $X$.

Theorem 1. If $T$ is in $\mathfrak{B}(\mathcal{H})$, then

$$
\operatorname{Ext}\left(W(T)^{-}\right) \subset W_{e}(T) \cup W(T)
$$

In particular,

$$
W(T)^{-}=\operatorname{convh}\left(W_{e}(T) \cup W(T)\right)
$$

Proof. Clearly $W(\alpha T+\beta)=\alpha W(T)+\beta$ for all $\alpha, \beta$ complex. Therefore, by rotation and translation, we can assume that $W(T)^{-}$is contained in the closed right half plane and $0 \in \operatorname{Ext}\left(W(T)^{-}\right)-W(T)$.

Then there exists a sequence $\left\{x_{n}\right\}_{1}^{\infty}$ of unit vectors of $\mathcal{H}$ such that $\left(T x_{n}, x_{n}\right) \rightarrow 0$. By the weak sequential compactness of the unit ball of $\mathcal{H}$ we can assume that $\left\{x_{n}\right\}_{1}^{\infty}$ converges weakly to $x \in \mathcal{H}$ with $\|x\| \leq 1$. We prove that $x$ is the 0 vector, and hence $0 \in W_{e}(T)$.

If $\|x\|=1$, then $x_{n} \rightarrow x$ strongly. But

$$
\begin{aligned}
|(T x, x)| & \leq\left|\left(T\left(x-x_{n}\right), x\right)\right|+\left|\left(T x_{n}, x-x_{n}\right)\right|+\left|\left(T x_{n^{\prime}} x_{n}\right)\right| \\
& \leq\left|\left(x-x_{n}, T^{*} x\right)\right|+\|T\|\left\|x-x_{n}\right\|+\left|\left(T x_{n^{\prime}} x_{n}\right)\right| \rightarrow 0 .
\end{aligned}
$$

Hence $(T x, x)=0$ and $0 \in W(T)$.

So assume $0<\|x\|<1$. Clearly the operator $\operatorname{Re} T$ is positive since $W(T)$ is contained in the closed right half plane. Then

$$
\left\|(\operatorname{Re} T)^{1 / 2} x_{n}\right\|^{2}=\left((\operatorname{Re} T) x_{n}, x_{n}\right)=\operatorname{Re}\left(T x_{n}, x_{n}\right) \rightarrow 0,
$$

so $\left\|(\operatorname{Re} T) x_{n}\right\| \rightarrow 0$. This clearly yields $\operatorname{Re}(T x, x)=0$, so $(T x, x)$ is purely imaginary. On the other hand,

$$
\left(T\left(x-x_{n}\right), x-x_{n}\right)=\left(T x, x-x_{n}\right)-\left(T x_{n}, x\right)+\left(T x_{n}, x_{n}\right) \rightarrow-(T x, x)
$$

and

$$
\left\|x-x_{n}\right\|^{2}=1-2 \operatorname{Re}\left(x-x_{n}, x\right)-\|x\|^{2},
$$

so $\left(T y_{n}, y_{n}\right) \rightarrow-(T x, x) /\left(1-\|x\|^{2}\right)$ where $y_{n}=\left(x-x_{n}\right) /\left\|x-x_{n}\right\|$. Thus we have produced nonzero purely imaginary points in $W(T)^{-}$which lie in the upper and lower half planes. However, this implies 0 is a nonextreme point of $W(T)^{-}$, thus completing the proof of the inclusion. The equality follows from the inclusion by the Krein-Milman theorem. 
We remark that an obvious equivalent form of the inclusion in Theorem 1 holds:

$$
\operatorname{Ext}\left(W(T)^{-}\right) \subset \operatorname{Ext}\left(W_{e}(T)\right) \cup \operatorname{Ext}(W(T))
$$

Corollary 1. $W(T)$ is closed if and only if $W_{\boldsymbol{e}}(T) \subset W(T)$. In particular, a compact operator has closed numerical range if and only if 0 is in its numerical range.

Proof. Necessity is obvious since $W_{e}(T) \subset W(T)^{-}$. If $W_{e}(T) \subset W(T)$, then $W(T)^{-}=\operatorname{convh}(W(T))=W(T)$.

The second statement follows from the first since the essential numerical range of a compact is $\{0\}$.

We remark that the second statement in Corollary 1 is an unpublished observation of A. Brown. It has also been observed by J. R. Giles and B. Sims [3].

Corollary 2. $W_{e}(T)=W(T)^{-}$if and only if $\operatorname{Ext}(W(T)) \subset W_{e}(T)$. Therefore, $W_{e}(T)=W(T)^{-}$if $W(T)$ bas no extreme points.

Proof. If $\operatorname{Ext}(W(T)) \subset W_{e}(T)$, then $\operatorname{Ext}\left(W(T)^{-}\right) \subset W_{e}(T) \subset W(T)^{-}$. Taking convex hulls, we obtain $W(T)^{-}=W_{e}(T)$. The reverse implication is obvious.

As a specific application of Corollary 2, we mention that $W_{e}\left(T_{\phi}\right)=$ $W\left(T_{\phi}\right)^{-}$for any Toeplitz operator $T_{\phi}$ on the Hardy space $H^{2}$ (see [4] for definitions). This follows from a the orem of E. Klein [6]: $\operatorname{Ext}\left(W\left(T_{\phi}\right)\right)$ is void.

For the next result, recall that $\lambda$ is called a corner of the convex set $C$ if $\lambda \in C$ and $C$ is contained in a sector with vertex $\lambda$ and opening less than $\pi$.

Corollary 3. If $W(T)^{-}$has a corner at the point $\lambda$, then $\lambda$ is in $W_{e}(T)$ or $\lambda$ is a reducing eigenvalue of finite multiplicity for $T$ wbich is an isolated point of $\sigma(T)$.

Proof. If $\lambda$ is not in $W_{e}(T)$, then $\lambda$ must be a corner of $W(T)$. By a theorem of W. Donoghue [1], $\lambda$ is an eigenvalue of $T$. A result of S. Hildebrandt [5] implies that the eigenspace corresponding to $\lambda$ reduces $T$. The rest of the proof follows from elementary Fredholm theory. $T-\lambda$ is Fredholm $\left(\lambda \notin \sigma_{e}(T)\right)$ of index 0 ( $\lambda$ reduces $\left.T\right)$; in particular, $\lambda$ has finite multiplicity. If $\lambda$ is not isolated, then there exist $\lambda_{n} \in \sigma(T)$ such that $\lambda_{n} \neq \lambda, \lambda_{n} \rightarrow \lambda$, and $T-\lambda_{n}$ is Fredholm of index 0 (this set is open). 
Hence, there exist $x_{n} \in \mathcal{H}$ such that $\left\|x_{n}\right\|=1, x_{n} \in \operatorname{rg}(T-\lambda)$ and $T x_{n}=\lambda_{n} x_{n}$. Thus the weak limits of $x_{n}$ lie in $\operatorname{rg}(T-\lambda) \cap \operatorname{ker}(T-\lambda)=0$. This yields a contradiction since $x_{n} \rightarrow 0$ weakly and $\left(T x_{n}, x_{n}\right) \rightarrow \lambda$ implies $\lambda \in W_{e}(T)$.

J. Anderson has communicated the following application of Corollary 3: A closed semidisk cannot be the numerical range of a compact operator. This fact is made evident in the next corollary, which is a mild extension of his idea.

We first give some terminology. Let $\lambda$ be a corner of $W(T)^{-}$and let $B_{\epsilon}(\lambda)=\{z \in \mathrm{C}:|z-\lambda|<\epsilon\}$. We shall say that $\lambda$ is lineal if there exists $\epsilon>0$ such that $B_{\epsilon}(\lambda) \cap \partial W(T)$ consists of line segments emanating from $\lambda_{\text {. }}$

Corollary 4. If $\lambda$ is a corner of $W(T)^{-}$and $\lambda \notin W_{e}(T)$ then $\lambda$ must be lineal. In particular, if $K$ is compact and $\lambda$ is a nonzero corner of $W(K)^{-}$, then $\lambda$ is lineal.

Proof. Corollary 3 implies $T=\lambda \oplus T_{1}$ with respect to $\operatorname{ker}(T-\lambda) \oplus$ $\operatorname{ker}(T-\lambda)^{\perp}$. Clearly $W\left(T_{1}\right) \subset W(T)$ and $W_{e}\left(T_{1}\right) \subset W_{e}(T)$. Hence $\lambda \notin W\left(T_{1}\right)^{-}$; otherwise, $\lambda$ would be a corner of $W\left(T_{1}\right)^{-}$not in $W_{e}\left(T_{1}\right)$ and hence an eigenvalue of $T_{1}$, thus producing a nonzero vector in $\operatorname{ker}(T-\lambda) \cap \operatorname{ker}(T-\lambda)^{\perp}$. Choose $\epsilon>0$ so that $B_{\epsilon}(\lambda) \cap W\left(T_{1}\right)^{-}=\varnothing$. But then (see $[4$, p. 113])

$$
W(T)^{-}=\left[\operatorname{convh}\left(\{\lambda\} \cup W\left(T_{1}\right)\right)\right]^{-}=\operatorname{convh}\left(\{\lambda\} \cup W\left(T_{1}\right)^{-}\right) .
$$

Clearly $B_{\epsilon}(\lambda) \cap \partial W(T)$ consists of line segments, so $\lambda$ is lineal. The second statement of the corollary obviously follows from the first.

For the next application recall that $T$ is hyponormal if $T^{*} T-T T^{*}$ is positive. $T$ is called seminormal if either $T$ or its adjoint $T^{*}$ is hyponormal.

Corollary 5. If $T$ is seminormal and $\lambda$ is an extreme point of $W(T)^{-}$, then either $\lambda \in W_{e}(T)$ or $\lambda$ is a reducing eigenvalue of finite multiplicity for $T$ which is an isolated point of $\sigma(T)$.

Proof. J. G. Stampfli has proved that extreme points of the numerical range of a seminormal operator are reducing eigenvalues [8]. The proof then proceeds as the one in Corollary 3.

The preceding allows a different proof of a perturbation result due to N. Salinas [7].

Corollary 6. If $T$ is seminormal, there exists a normal and compact operator $K$ such that $T$ commutes with $K$ and $W(T+K)^{-}=W_{e}(T)$. 
Proof. Corollary 5 clearly implies that if $T$ is seminormal,

$$
W(T)^{-}=\operatorname{convh}\left(W_{e}(T) \cup \Pi_{\text {oor }}(T)\right),
$$

where $\Pi_{\text {oor }}(T)$ denotes the countable set of isolated reducing eigenvalues of finite multiplicity for $T$. $T$ can be written as $T=T_{1} \oplus \lambda_{1} \oplus \lambda_{2} \oplus \ldots$ with $\left\{\lambda_{n}\right\}=\Pi_{\text {oor }}(T)-W_{e}(T)$ and $T_{1}$ seminormal. If $\left\{\lambda_{n}\right\}$ is finite, choose $\left\{\mu_{n}\right\} \subset$ $W_{e}(T)$; otherwise, choose $\left\{\mu_{n}\right\} \subset W_{e}(T)$ so that $\left|\mu_{n}-\lambda_{n}\right| \rightarrow 0$ (the cluster set of $\left\{\lambda_{n}\right\}$ lies in $\partial W_{e}(T)$, so $\left\{\mu_{n}\right\}$ exists). In either case, let $K$ be the compact normal operator $0 \oplus\left(\mu_{1}-\lambda_{1}\right) \oplus\left(\mu_{2}-\lambda_{2}\right) \oplus \ldots$ Clearly $T$ commutes with $K, T+K$ is seminormal, and $\Pi_{\text {oor }}(T+K) \subset W_{e}(T)=W_{e}(T+K)$. Hence

$$
W(T+K)^{-}=\operatorname{convh}\left(W_{e}(T+K) \cup \Pi_{\text {oor }}(T+K)\right)=\operatorname{convh}\left(W_{e}(T)\right)=W_{e}(T) .
$$

The following lemma is a restatement of Lemma 1 in [8].

Lemma. Let $\operatorname{Re} W(T) \geq 0$ and let 9 denote the imaginary axis. Then $W(T) \cap \mathcal{I}=\{(T x, x):\|x\|=1$ and $x \in \operatorname{ker}(\operatorname{Re} T)\}$.

An extended version of Theorem 1 can now be obtained.

The orem 2. For any $T$ in $\mathfrak{B}(\mathcal{H})$,

$$
W(T)^{-}=\left(W_{e}(T) \cup W(T)\right) \dot{\cup}(T),
$$

where $\mathfrak{L}(T)$ is a countable disjoint union of open complex line segments each of which has one endpoint in $\operatorname{Ext}(W(T))-W_{e}(T)$ and the other in $\operatorname{Ext}\left(W_{e}(T)\right)-W(T) .(\dot{u}$ denotes disjoint union. $)$

Proof. Suppose $\lambda \in W(T)^{-}$and $\lambda \notin W_{e}(T) \cup W(T)$. Then by Theorem 1 , $\lambda$ is a nonextreme boundary point of $W(T)^{-}$. Hence there exists a line $l$ through $\lambda$ such that $W(T)^{-}$lies on one side of $l$ and such that $W(T)^{-} \cap l$ $=[\alpha, \beta]$ with $\alpha \neq \beta$. Clearly. $\alpha, \beta \in \operatorname{Ext}\left(W(T)^{-}\right) \subset W(T) \cup W_{e}(T)$, so we can assume $\alpha \in \operatorname{Ext}(W(T))-W_{e}(T)$ and $\beta \in \operatorname{Ext}\left(W_{e}(T)\right)-W(T)$. But since $W_{e}(T)$ is closed, $W_{e}(T) \cap[\lambda, \beta]$ is of the form $[\eta, \beta]$ with $\eta \epsilon$ $\operatorname{Ext}\left(W_{e}(T)\right)-W(T)$. It remains to show that $W(T) \cap[\alpha, \lambda]$ is of the form $[\alpha, \mu]$, so that then $\lambda \in(\mu, \eta) \subset \mathcal{L}(T)$ with $\mu, \eta$ as required.

Without loss of generality we can assume $\operatorname{Re} W(T) \geq 0, \eta=0$, the segment $[\alpha, \lambda]$ lies in the positive imaginary axis with $|\alpha|>|\lambda|$, and $W_{e}(T) \cap g$ is contained in the nonpositive imaginary axis. If $\operatorname{ker}(\operatorname{Re} T)$ is infinite dimensional, choose an orthonormal basis $\left\{e_{n}\right\}_{1}^{\infty}$ for $\operatorname{ker}(\operatorname{Re} T)$. The lemma then implies $\left(T e_{n}, e_{n}\right) \in W(T) \cap[\alpha, \lambda]$ for every $n$. By passing to a 
subsequence, if necessary, $\left(T e_{n}, e_{n}\right)$ converges to a number on the positive imaginary axis. But this number must lie in $W_{e}(T)$, which is impossible. Hence, $\operatorname{ker}(\operatorname{Re} T)$ must be finite dimensional. Again by the lemma, $W(T) \cap$ $[\alpha, \lambda]=W(T) \cap \mathcal{G}=W\left(\left.P T\right|_{\mathrm{rg}(P)}\right)$, where $P$ is the orthogonal projection onto $\operatorname{ker}(\operatorname{Re} T)$ with range $\mathrm{rg}(P)$. Since the numerical range of a finite dimensional operator is closed, $W(T) \cap[\alpha, \lambda]$ is of the desired form.

Corollary 7. The numerical range of a compact operator contains all its boundary points, with the possible exception of one or two (perbaps degenerate) complex line segments of the form $[0, a)$.

Examples: The convex set convh $((0,1] \cup(i, 2 i])$ cannot be the numerical range of a compact operator $K$ because $\partial W(K)-W(K)$ would then be the closed line segment $[0, i]$. It is easy, however, to construct a diagonal compact operator whose numerical range is $\operatorname{convh}((0,1] \cup[i, 2 i])$ : let $D e_{1}=i e_{1}, D e_{2}=2 i e_{2}$, and $D e_{n}=(n-2)^{-1} e_{n}$ for $n \geq 3$ where $\left\{e_{n}\right\}_{1}^{\infty}$ is an orthonormal basis for $\mathcal{H}$.

Acknowledgement. The author wishes to thank J. P. Williams for his helpful discussions and suggestions.

\section{REFERENCES}

1. W. F. Donoghue, Jr., On the numerical range of a bounded operator, Michigan Math. J. 4 (1957), 261-263. MR 20\#2622.

2. P. A. Fillmore, J. G. Stampfli and J. P. Williams, On the essential numerical range, the essential spectrum, and a problem of Halmos, Acta Sci. Math. (Szeged) 33 (1972), 179-192. MR 48 \#896.

3. J. R. Giles and B. Sims, On the numerical range of compact operators on Hilbert space (preprint).

4. P. R. Halmos, A Hilbert space problem book, Van Nostrand, Princeton, N. J., 1967. MR $34 \# 8178$.

5. S. Hildebrandt, Über den numerischen Wertebereich eines Operators, Math. Ann。163 (1966), 230-247. MR 34 \#613.

6. E. Klein, Toeplitz operators and Weyl spectra, Dissertation, Northwestern University, 1971.

7. N. Salinas, Operators with essentially disconnected spectra, Acta Sci。 Math. (Szeged) 33 (1972), 193-206.

8. J.G. Stampfli, Extreme points of the numerical range of a hyponormal operator, Michigan Math. J. 13 (1966), 87-89. MR 32 \#551。

9. J. G. Stampfli and J. P. Williams, Growth conditions and the numerical range in a Banach algebra, Tohoku Math. J. (2) 20 (1968), 417-424. MR 39\#4674。

DEPARTMENT OF MATHEMATICS, MARSHALL UNIVERSITY, HUNTING TON, WEST VIRGINIA 25701 